\title{
Viscoelastic properties of the small intestinal and caecal contents of the chicken
}

\author{
T. Takahashi ${ }^{1}$, M. Goto ${ }^{2}$ and T. Sakata ${ }^{3 *}$ \\ ${ }^{1}$ Laboratory of Animal Nutrition, Faculty of Agriculture, Okayama University, Tsushimanaka 1-1-1, \\ Okayama 700-8530, Japan \\ ${ }^{2}$ Faculty of Bioresources, Mie University, Kamihama-cho 1515, Tsu 514-8507, Japan \\ ${ }^{3}$ Department of Basic Sciences, Ishinomaki Senshu University, Ishinomaki 986-8580, Japan
}

(Received 16 May 2003 - Revised 30 January 2004 - Accepted 16 February 2004)

\begin{abstract}
We measured the coefficients of viscosity, shear rates and shear stresses of chicken small intestinal and caecal contents, including solid particles, using a tube-flow viscometer. The coefficients of viscosity of chicken small intestinal and caecal contents were correlated negatively with their shear rates, a characteristic typical of non-Newtonian fluids. The coefficient of viscosity of the small intestinal contents was lower than that of the caecal contents at a shear rate of $1 \mathrm{~s}^{-1}$. Chicken caecal contents were more viscous than pig caecal contents. The exponential relationship between shear stress and shear rate showed that chicken small intestinal and caecal contents had an apparent Herschel-Bulkley fluid nature. These results indicate that solid particles, including uric acid crystals, are mainly responsible for the viscosity of the digesta in the chicken.
\end{abstract}

Broiler: Intestinal contents: Viscoelasticity: Flow behaviour

The viscosity of the intestinal contents can affect enzymic reactions (Hasinoff et al. 1987) and nutrient absorption (Antoon \& Kirsch, 1982), and should define the flow behaviour (Cheremisinoff, 1986) in the lumen of the intestine. The viscosity of the gut contents suppresses the absorption of nutrients in the small intestine of poultry (van der Klis et al. 1993).

Particle crowding depresses the diffusion rate of substrates in fluids (García-Pérez et al. 1999); the diffusion rate of a nutrient in the gut contents should positively correlate with the reaction rate (Hasinoff et al. 1987). Therefore, particle crowding should play a significant role in the digestion and absorption of nutrients in the gut lumen.

The caecum of the chicken accumulates fine particles, including uric acid (Karasawa, 1989), from which amino acids and protein are synthesized microbiologically and absorbed into the body pool (Klasing, 2000). Accordingly, the viscosity and flow behaviour of the caecal contents should affect the synthesis and absorption of amino acids in this organ.

To date, the viscosity of intestinal contents has been measured only after the complete removal of solid particles by centrifugation (Bedford et al. 1990; Zubair et al. 1996; Razdan \& Pettersson, 1996; Jaroni et al. 1999). However, solid particles are the main determinant of the viscosity of pig caecal contents (Takahashi \& Sakata, 2002). Therefore, it is important to measure the viscosity of whole digesta to understand the processes of mixing, digestion and absorption within the chicken gut lumen. Accordingly, the present study describes the viscoelastic properties of chicken small intestinal and caecal contents, including solid particles. We also compared the viscosity of digesta with and without particles to estimate the contribution of particles to the viscosity of digesta in the chicken.

\section{Materials and methods}

\section{Sample}

We collected approximately $700 \mathrm{ml}$ whole small intestinal or whole caecal contents from approximately 200 healthy broiler chickens, each weighing approximately $2.7 \mathrm{~kg}$ (48-54 d old). They were fed on Miyagi Shiage (Marubeni Shiryo Corporation, Tokyo, Japan) diet for at least $10 \mathrm{~d}$; the diet included $(\mathrm{g} / \mathrm{kg})$ : maize 280 , grain sorghum 361 , soyabean meal 208, rapeseed meal 30, maize gluten meal 6 , fish meal 9, chicken meal 11, and calcium carbonate plus feed additive 95, with no antibiotics. They were slaughtered at Heisei Farm Corporation (Miyagi branch, Kanan-cho, Miyagi, Japan), after being starved for several hours. The diet included $120 \mathrm{~g}$ particles $>2.54 \mathrm{~mm} / \mathrm{kg}, 732 \mathrm{~g}$ particles $1.06-2.54 \mathrm{~mm} / \mathrm{kg}$ and $148 \mathrm{~g}$ particles $<1.06 \mathrm{~mm} / \mathrm{kg}$. The small intestinal contents, including the whole digesta in the duodenum, jejunum and ileum, were sampled by free draining through a cut-off end of the small intestine. The caecal contents were sampled as drainage after cutting 
the intestinal wall transversely and longitudinally, both to eliminate any possibility of contamination with epithelial cells and to minimize mechanical stimuli that might stimulate mucus secretion. Such sampling was replicated twice to measure the viscosity of the small intestinal contents (n 2) and three times for the caecal contents (n 3). All the chickens were healthy and had passed a veterinary inspection by the local authority (Senpoku Meat Inspection Centre, Yoneyama-machi, Miyagi, Japan).

\section{Measurement of the density and DM of gut contents}

We measured the volume and weight of the intestinal contents at $41^{\circ} \mathrm{C}$ after each series of viscometry measurements to calculate their density.

We also determined the DM in the samples as the weight difference before and after drying the contents in a vacuum desiccator (IUCHI Corporation, Osaka, Japan), at approximately $21 \mathrm{kPa}$ at room temperature for $72 \mathrm{~h}$.

\section{Viscometry}

We measured the viscosity of chicken small intestinal and caecal contents containing solid particles using the HagenPoiseuill law (Darby, 1988). For this purpose, we built a tube-flow viscometer using glass tubes (Fig. 1; Takahashi \& Sakata, 2002). We used glass tubes of 10 and $21 \mathrm{~mm}$ inner diameter and $1000 \mathrm{~mm}$ long for the small intestinal and caecal contents respectively. Since the viscous properties are theoretically unaffected by the inner diameter of the glass tube (Fox \& McDonald, 1985), we chose the inner diameters so that the gut contents would fill the tube, but would neither plug it nor slip on its wall.

We calculated the coefficient of viscosity from the volume flow rate and pressure drop of the small intestinal and caecal contents through glass tubes of known inner diameter and length. The pressure drop was the difference in pressure between the upper level of the glass tube and its lower exit. The pressure drop was controlled by changing the difference in height between the upper level of the contents in the reservoir and the lower exit of the glass tube (Fig. 1). Two thermostats kept the temperature of the gut contents in the reservoir and glass tube at $41 \pm 1^{\circ} \mathrm{C}$.

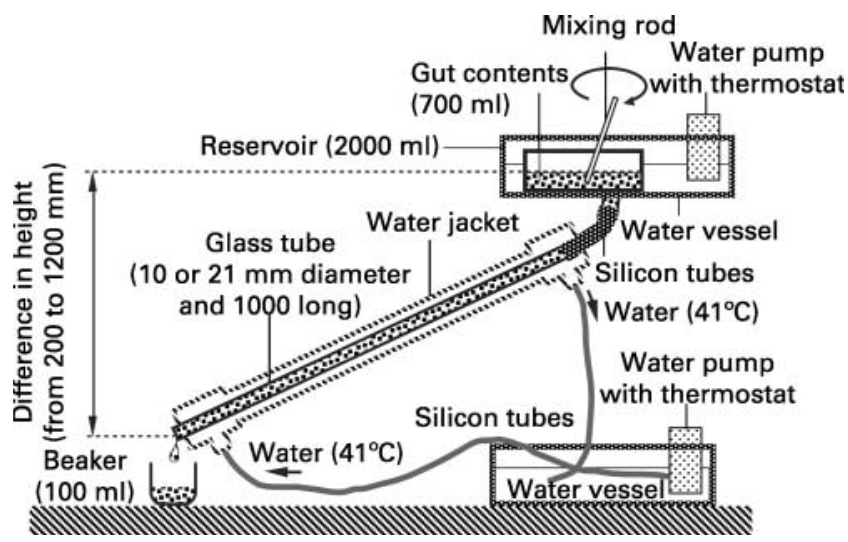

Fig. 1. Tube-flow viscometry for chicken small intestinal and caecal contents.

\section{Measuring the volume flow rate}

We poured approximately $700 \mathrm{ml}$ gut contents into the reservoir. Then, we measured the volume flow rate at pressure drops of 4800, 3800, 2900 and $1900 \mathrm{~Pa}$ for small intestinal contents and 12000, 11000, 9700 and $8700 \mathrm{~Pa}$ for caecal contents, until the drained volume reached approximately $50 \mathrm{ml}$. The drained contents were returned to the reservoir and reused in the same measurement series. The gut contents in the reservoir were mixed manually with glass rod throughout the measurement. The series of measurements of the small intestinal and caecal contents were replicated twice and three times respectively, using different samples.

\section{Calculating viscosity, shear stress and shear rate}

The local flow rate of a fluid shows a velocity gradient in the flow in a tube (Borghesani, 1988). If a fluid flows in a tube, the local flow rate at the periphery is lower than that in the centre (Borghesani, 1988). Accordingly, there is a velocity gradient in the flow (Lentle et al. 2002). This velocity gradient is defined as the shear rate. Generally, a high flow rate accompanies a high shear rate in the tube (Borghesani, 1988). Shear stress is the pressure needed to make a fluid move and is expressed as force per unit area (Borghesani, 1988). We calculated the shear stress, shear rate and coefficient of viscosity of the small intestinal or caecal contents (Darby, 1988):

$$
\text { shear stress } \begin{aligned}
(\mathrm{Pa})= & (\text { pressure drop }(\mathrm{Pa}) \\
& \times \text { radius of glass tube }(\mathrm{m})) / \\
& (2 \times \text { length of glass tube }(\mathrm{m})),
\end{aligned}
$$

pressure drop $(\mathrm{Pa})=($ difference in height $(\mathrm{m}))$

$$
\times\left(\text { density }\left(\mathrm{kg} / \mathrm{m}^{3}\right)\right) \times 9.8\left(\mathrm{~m} / \mathrm{s}^{2}\right) \text {, }
$$

and

$$
\begin{aligned}
\text { shear rate }\left(\mathrm{s}^{-1}\right)= & \left(4 \times \text { volume flow rate }\left(\mathrm{m}^{3} / \mathrm{s}\right)\right) / \\
& \left(\pi \times(\text { radius of glass tube }(\mathrm{m}))^{3}\right) \\
& \times((3 \times(\mathrm{N}+1)) /(4 \times \mathrm{N})),
\end{aligned}
$$

where $\mathrm{N}$ is the slope of the least squares linear regression equation between log (pressure drop $(\mathrm{Pa})$ ) (dependent variable) and $\log$ (volume flow rate $\left(\mathrm{m}^{3} / \mathrm{s}\right)$ ) (independent variable). Finally,

$$
\begin{aligned}
& \text { coefficient of viscosity }(\mathrm{Pa} \cdot \mathrm{s}) \\
& =\text { shear stress }(\mathrm{Pa}) / \text { shear rate }\left(\mathrm{s}^{-1}\right) \text {. }
\end{aligned}
$$

Potential effect of fermentation during the measurement of gut content viscosity

A series of viscometry measurements took approximately $3 \mathrm{~h}$. Hence, we tested the difference in the coefficient of viscosity of the small intestinal or caecal contents between the first and last measurements. This was done at pressure drops of 4800 and $12000 \mathrm{~Pa}$ for the small intestinal and caecal contents respectively to evaluate the effect of potential fermentation during the series of measurements on the viscosity of the contents. 


\section{Statistical analyses}

The results were expressed as mean values with their standard errors. There was no significant difference in the coefficients of viscosity of the small intestinal or caecal contents among replicates in preliminary analysis of covariance (covariate: shear rate) (Zar, 1999) (small intestinal contents $P=0 \cdot 30$, error df 8 ; caecal contents $P=0.57$, error df 5 ). We therefore calculated regression equations for the small intestinal or caecal contents using data pooled for all replicates.

The coefficient of viscosity of a non-Newtonian fluid depends on its shear rate (Cheremisinoff, 1986). Since we did not measure the viscosity of the small intestinal and caecal contents at the same shear rate, we estimated the viscosity of the small intestinal and caecal contents at a shear rate of $1 \mathrm{~s}^{-1}$ using a power regression equation of viscosity $v$. shear rate from pooled data after jack-knife resampling (Sokal \& Rohlf, 1995). Then, we tested the difference in viscosity between small intestinal and caecal contents using one-way ANOVA (Zar, 1999).

The difference in the coefficient of viscosity of the small intestinal or caecal contents between the first and last trials, at the same pressure drop, was tested using one-way ANOVA (Zar, 1999).

The difference between mean values was considered significant when the error probability was $<0.05$. We used JMP version 5 software (SAS Institute Japan, Tokyo, Japan) for the statistical analyses

\section{Results}

The densities of the small intestinal and caecal contents with particles were 940 and $1000 \mathrm{~kg} / \mathrm{m}^{3}(n$ 2) and 1030 (SE 2) $\mathrm{kg} / \mathrm{m}^{3}$ (n 3) respectively. Chicken small intestinal (a)

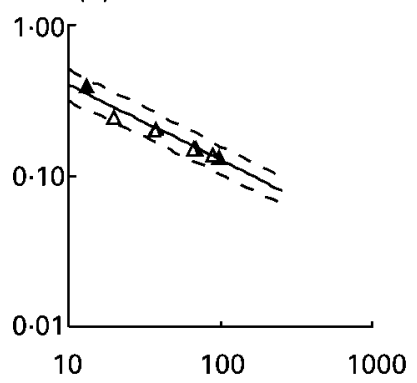

(b)

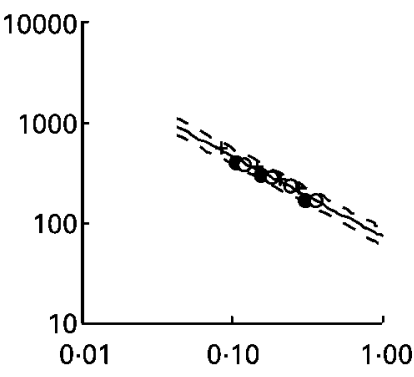

Shear rate $\left(\mathrm{s}^{-1}\right)$

Fig. 2. Coefficient of viscosity $v$. the shear rate of chicken smal intestinal $((\mathrm{a}) ; y=(1.2 \pm 0.2) x, \Delta$, replication $1 ; \Delta$ replication 2$)$ and caecal contents $\left((\mathrm{b}) ; y=(74 \pm 6) x^{-(0.79 \pm 0.04)}\right) ; \circ$, replication $1 ;+$ replication 2 ; $\bullet$, replication 3$)$ at $41^{\circ} \mathrm{C}$. ---, $95 \%$ confidence limit for the power regression equation $(P<0.05)$. The viscosity of these gut contents was measured using the flow behaviour through a glass tube $10 \mathrm{~mm}$ in diameter and $1 \mathrm{~m}$ long, with 4800,3800, 2900, and $1900 \mathrm{~Pa}$ pressure drops, to measure the viscosity of small intestinal contents, or a tube $21 \mathrm{~mm}$ in diameter and $1 \mathrm{~m}$ long with 12000 , 11000,9700 and $8700 \mathrm{~Pa}$ pressure drops to measure the viscosity of caecal contents (logarithmic scale). For details of procedures, see Fig. 1 and p. 868. Note the different scales on the $y$-axes in the left and right panels. or caecal contents contained 162 and $159 \mathrm{~g} \mathrm{DM} / \mathrm{kg}(n$ 2) and 188 (SE 8) g DM/kg (n 3) respectively.

There was no significant difference in the coefficients of viscosity of the small intestinal or caecal contents between the first and last measurements at the same pressure drop (small intestinal contents $P=1 \cdot 0$, error df 1 ; caecal contents $P=0 \cdot 8$, error df 3 )

The coefficient of viscosity decreased as the shear rate increased for the small intestinal and caecal contents (Fig. 2).

The estimated coefficient of viscosity at a shear rate of $1 \mathrm{~s}^{-1}$ for the small intestinal contents $(1 \cdot 2$ (SE 0.1) Pa.s, $n$ 4) was considerably lower than that of the caecal contents (73 (SE 1.1) Pa.s, $n$ 4) $(P<0 \cdot 001$, error df 6).

The shear stress and shear rate of both contents showed a power correlation with a positive shear stress value at a shear rate $0 \mathrm{~s}^{-1}$ (Fig. 3).

\section{Discussion}

\section{Viscometry}

We confirmed the validity of our tube-flow viscometry method by comparing the viscosity of model digesta, i.e. a suspension of cellulose in carboxymethyl cellulose solution, measured using our method (the tube of $10 \mathrm{~mm}$ ) and using a cone plate viscometer (TV-20; Toki Sangyo Co., Ltd, Tokyo, Japan) ( $\mathrm{T}$ Takahashi, unpublished results). The viscous properties measured using the two methods agreed well $(P=0 \cdot 17$, error df 8 , analysis of covariance (covariate: shear rate) (Zar, 1999)). We had already confirmed in our previous study that the viscous property was independent of the inner diameter of the glass tubes (Takahashi \& Sakata, 2002). Therefore, it was valid to compare the viscosity of small intestinal and caecal contents measured using glass tubes with different diameters.

\section{Effect of potential fermentation}

There was no significant difference in the coefficient of viscosity of the small intestinal or caecal contents between (a)

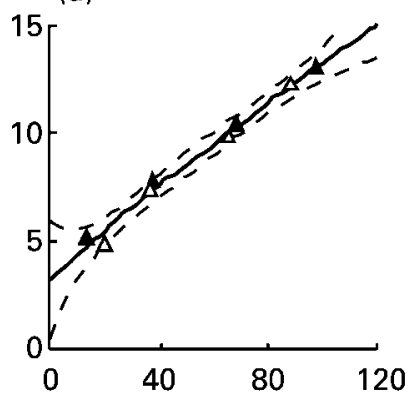

(b)

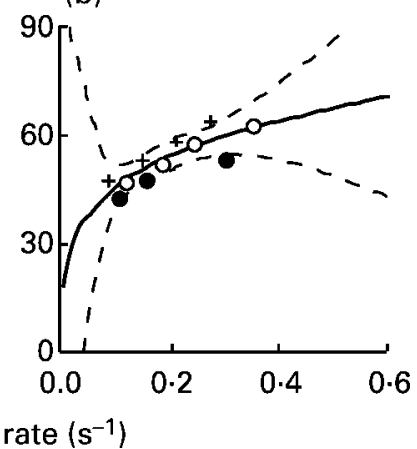

Fig. 3. Shear stress $v$. against shear rate of chicken small intestinal $\left((\mathrm{a}) ; y=(0.16 \pm 0.017) x^{(0.90 \pm 0.21)}+(3.2 \pm 1.1) ; \Delta\right.$, replication $1 ; \Delta$, replication 2$)$ and caecal contents $\left((\mathrm{b}), y=(74 \pm 175) x^{(0.21 \pm 1 \cdot 15)} ; \circ\right.$, replication $1 ;+$, replication 2 ; •, replication 3$).---, 95 \%$ confidence limit for the regression equation $(P<0.001)$. For details of procedures, see Fig. 1 and p. 868. Note the positive $y$-intercept of the regression line. 
the first and last measurements, at the same pressure drop (see p. 869). This suggests that possible fermentation or mixing of the contents during the series of measurements did not affect their viscosity.

\section{Viscosity of the chicken intestinal contents}

The coefficient of viscosity decreased with an increase in shear rate (Fig. 2). This characteristic is typical of nonNewtonian fluids (Cheremisinoff, 1986), such as pig caecal contents (Takahashi \& Sakata, 2002).

The removal of solid particles reduced the coefficient of viscosity of pig caecal contents considerably and made the contents into a typical Newtonian fluid, i.e. the shear rate did not influence the coefficient of viscosity (Takahashi et al. 2001). This should also apply to chicken gut contents. The published coefficient of viscosity of chicken small intestinal and caecal contents, after removing the solid particles by centrifugation, is $0 \cdot 84-1.4 \mathrm{mPa} \cdot \mathrm{s}$ at a shear rate of $2.3 \mathrm{~s}^{-1}$ (centrifugation at $12000 \mathrm{~g}$ ) (Razdan \& Pettersson, 1996 ), or $3.6-5.8 \mathrm{mPa} \cdot \mathrm{s}$ at the shear rate of $45 \mathrm{~s}^{-1}$ (centrifugation at $15000 \mathrm{~g}$ ) (Zubair et al. 1996). These values are approximately 500-1000 times lower than our present results (Fig. 2).

The difference between our findings and previously published viscosities might reflect the use of different centrifugation forces. Accordingly, we compared the viscosity of the supernatant fraction of chicken small intestinal contents after centrifugation at 12000,15000 and $16000 \mathrm{~g}$ using a cone-plate viscometer, with a CP-40 cone (LVDV-I; Brookfield Engineering Laboratories, Inc., Middleboro, MA, USA), at shear rates of $150.0,75 \cdot 0,37.5,30.0$ and $18.8 \mathrm{~s}^{-1}$ (n 3) ( $\mathrm{T}$ Takahashi, unpublished results). There was no significant difference in the coefficient of viscosity of the small intestinal contents after centrifugation at 12000,15000 or $16000 \mathrm{~g}$ by two-way ANOVA (Zar, 1999) (centrifugation $P=0 \cdot 81$, shear rate $P=0 \cdot 87$, interaction between centrifugation and shear rate $P=0.99$, error $\mathrm{df} 30$ ). The coefficient of viscosity of the small intestinal contents after centrifugation was $2 \cdot 1$ (SE $0 \cdot 1) \mathrm{mPa} \cdot \mathrm{s}$ ( $n$ 45), which did not depend on the shear rate $(P=0.58$, error df 41). This, together with the published values, confirms the considerable contribution of solid particles to the viscosity of chicken intestinal contents. This suggests that the ingestion of solid particles, such as cellulose, may elevate the viscosity of chicken gut contents, as it does in rats (Takahashi et al. 2003).

Chicken small intestinal and caecal contents contained 160 and $188 \mathrm{~g} / \mathrm{kg}$ DM respectively (see p. 869). The supernatant fraction in previous studies (Hoskins \& Zamcheck, 1968; Razdan \& Pettersson, 1996; Zubair et al. 1996) probably included mucin after centrifugation at $16000 \mathrm{~g}$ (Hoskins \& Zamcheck, 1968). We estimated the mucin content in the supernatant fraction after centrifugation at 12000,15000 and 16000 gby measuring hexose, using the phenol-sulfuric acid method (Dubois et al. 1956). There was no significant difference in the hexose concentration $(10.0(\mathrm{SE} 0.7) \mathrm{mg} / \mathrm{l}, n 9)$ in the supernatant fraction of the small intestinal contents after centrifugation at 12000,15000 and $16000 \mathrm{~g}$ by ANOVA (Zar, 1999) (centrifugation $P=0.97$, error df 9). Therefore, the mucin was probably retained in the supernatant fraction after centrifuging the chicken caecal contents in this and in previous studies (Hoskins \& Zamcheck, 1968; Razdan \& Pettersson, 1996; Zubair et al. 1996). Accordingly, the far greater viscosity of the intact caecal contents in our present results compared with chicken small intestinal or pig caecal contents is due to solid particles, not mucin. In this regard, it is not feasible to speculate the diffusion rate across the gut contents, or the mobility of normal gut contents, based on the viscosity of the supernatant fraction alone.

\section{Viscoelastic characteristics of chicken intestinal contents}

The relationship between the shear stress $(y)$ and shear rate $(x)$ of small intestinal and caecal contents can be fitted to a power model with a positive $y$-intercept $\left(y=a x^{n}+c\right)$ (Fig. 3). This is a characteristic property of a HerschelBulkley fluid (Zimeri \& Kokini, 2003), and does not contradict the properties of the digesta of the forestomach in the tammar (Macropus eugenii) and the parma (Macropus parma) wallaby (Lentle et al. 2002). Yoghurt (Yoon \& McCarthy, 2002), mud (Huang \& Garcia, 1998) and fresh concrete (de Larrard et al. 1998) are typical Herschel-Bulkley fluids. The $y$-intercept of the regression equation (Fig. 3) is called the 'yield stress' (Zimeri \& Kokini, 2003). Herschel-Bulkley fluids behave as solids when the shear stress is smaller than the yield stress, and behave as fluids when the shear stress is higher (Zimeri \& Kokini, 2003). In the present study, the yield stresses for intact small intestinal and caecal contents were estimated to be 3.2 (SE 1.1) and 6.1 (SE 190) Pa respectively (Fig. 3).

\section{Comparison of chicken with pig caecal contents}

The coefficient of viscosity of chicken caecal contents $(82 \mathrm{~Pa} \cdot \mathrm{s})$ at a shear rate of $1 \mathrm{~s}^{-1}$ (Fig. 2) was approximately 22 times greater than that of pig caecal contents (3.7 Pa.s) (Takahashi et al. 2001). Therefore, chicken caecal contents are more viscous and should have more resistance to movement than pig caecal contents. This suggests much poorer mixing of the substrate and bacteria in the chicken caecum than in the pig caecum.

\section{Relationship between solid particles and viscosity}

As stated earlier, the presence of DM (i.e. solid particles) in the gut contents should elevate their viscosity (Jeffrey \& Acrivos, 1976; Takahashi \& Sakata, 2002). The DM levels of pig caecal (Takahashi et al. 2001), chicken small intestinal, and chicken caecal contents were 140, 160 and $188 \mathrm{~g} / \mathrm{kg}$ respectively (see p. 000). However, the viscosity of chicken caecal contents $(82.0 \mathrm{~Pa} \cdot \mathrm{s})$ at a shear rate of $1 \mathrm{~s}^{-1}$ was far greater than that of chicken small intestinal contents $(3 \cdot 8 \mathrm{~Pa} \cdot \mathrm{s})$ or pig caecal contents (3.7 Pa.s). This suggests that the viscosity of the gut contents is not a simple function of the DM level, as suggested in a study using coarse settling suspensions (Clarke, 1967). The size, shape, surface area and surface characteristics of the particles in gut contents should affect their viscosity (Saraf \& Khullar, 1975). 
The caecum of the chicken selectively collects fine particles and accommodates exclusively fine particles, such as bacteria and uric acid, which are excreted into the cloaca (Clemens et al. 1975). In avian caecal contents, 970 (SE 30) g particles $/ \mathrm{kg}$ are $<0.2 \mathrm{~mm}$, while 550 (SE 120) $\mathrm{g} / \mathrm{kg}$ avian small intestinal contents are $<0.2 \mathrm{~mm}$ (Björnhag \& Sperber, 1977) and 600 (SE 78) g/kg pig caecal contents are particles $<1 \mathrm{~mm}$ (Takahashi et al. 2001). Therefore, chicken caecal contents include a larger proportion of smaller particles than do chicken small intestinal or pig caecal contents. The inclusion of a large quantity of fine particles in chicken caecal contents may be responsible for their higher viscosity (Borghesani, 1988).

Uric acid in the colon exists as a colloidal suspension of small spherical bodies that range in diameter from 0.5 to $1.3 \mu \mathrm{m}$ (Braun, 1999), and originate from the cloaca (Karasawa, 1989). Accordingly, one of the outstanding features of chicken caecal contents is the existence of very small particles of uric acid. The caecal contents include uric acid as $80 \mathrm{mg} / \mathrm{g}$ DM (Isshiki, 1980). This amount of very small uric acid particles in chicken caecal contents might be responsible for their viscosity.

In conclusion, we showed that solid particles elevate the viscosities of chicken small intestinal and caecal contents. Previous studies have shown that dietary solid particles elevate the viscosities of digesta in pigs (Takahashi \& Sakata, 2002) and rats (Takahashi et al. 2003). The ingestion of solid particles, such as fibre, should elevate the viscosity of digesta in chicken, as in pigs and rats. Considering the effects of the viscosity of digesta on digestive enzyme reactions (Hasinoff et al. 1987), nutrient diffusion behaviour (Takahashi \& Sakata, 2002), the absorption rate (Jaroni et al. 1999) and the mixing of digesta in the lumen (Lentle et al. 2002), the viscosity of digesta with particles should help to reveal the processes involved in digestion and absorption.

\section{Acknowledgements}

We thank Mr M. Suzuki of The Heisei Farm Corporation and Mr K. Sasaki of The Livestock Research Division, Mie Prefecture Science and Technology Promotion Centre, for their generous help with sampling the chicken gut contents. We also thank E. Suzuki of Kyowa Kagaku Corporation and T. Mishima of The Faculty of Bioresources, Mie University, for their kind advice on viscometry and the phenol-sulfuric acid method.

\section{References}

Antoon BC \& Kirsch JF (1982) Investigation of diffusion-limited rates of chymotrypsin reactions by viscosity variation. Biochemistry 21, 1302-1307.

Bedford MR, Classen HL \& Campbell GL (1990) The effect of pelleting, salt, and pentosanase on the viscosity of intestinal contents and the performance of broilers fed rye. Poult Sci 70, $1571-1577$.

Björnhag G \& Sperber I (1977) Transport of various food components through the digestive tract of turkeys, geese and guinea fowl. Swed J Agric Res 7, 57-66.
Borghesani AF (1988) Non-Newtonian flow behavior of coalfuel oil suspensions. In Encyclopedia of Fluid Mechanics. Volume 7: Rheology and Non-Newtonian Flows, pp. 89-134 [NP Cheremisinoff, editor]. Houston, TX: Gulf Publishing Company.

Braun EJ (1999) Integration of renal and gastrointestinal function. J Exp Zool 283, 495-499.

Cheremisinoff NP (1986) Properties and concepts of single fluid flows. In Encyclopedia of Fluid Mechanics, Volume 1: Flow Phenomena and Measurement, pp. 285-351 [NP Cheremisinoff, editor]. Houston, TX: Gulf Publishing Company.

Clarke B (1967) Rheology of coarse settling suspensions. Trans Inst Chem Eng 45, T251-T256.

Clemens ET, Stevens CE \& Southworth M (1975) Site of organic acid production and pattern of digesta movement in the gastrointestinal tract of geese. $J$ Nutr 105, 1341-1350.

Darby R (1988) Laminar flow and turbulent pipe flows of nonNewtonian fluids. In Encyclopedia of Fluid Mechanics. Volume 7: Rheology and Non-Newtonian Flows, pp. 19-54 [NP Cheremisinoff, editor]. Houston, TX: Gulf Publishing Company.

De Larrard F, Ferraris CF \& Sedran T (1998) Fresh concrete: A Herschel-Bulkley material. Mater Struct 31, 494-498.

Dubois M, Gilles KA, Hamilton JK, Rebers PA \& Smith F (1956) Colorimetric method for determination of sugars and related substances. Anal Chem 28, 350-356.

Fox RW \& McDonald AT (1985) Internal incompressible viscous flow. In Introduction to Fluid Mechanics, 3rd ed., pp. 331-388. New York: John Wiley \& Sons.

García-Pérez AI, López-Beltrán EA, Klüner P, Luque J, Ballesteros P \& Cerdán S (1999) Molecular crowding and viscosity as determinants of translational diffusion of metabolites in subcellular organelles. Arch Biochem Biophys 362, 329-338.

Hasinoff BB, Dreher R \& Davey JP (1987) The association reaction of yeast alcohol dehydrogenase with coenzyme is partly diffusion-controlled in solvents of increased viscosity. Biochim Biophys Acta 911, 53-58.

Hoskins CL \& Zamcheck N (1968) Bacterial degradation of gastrointestinal mucins. Gastroenterology 54, 210-217.

Huang X \& Garcia MH (1998) A Herschel-Bulkley model for mud flow down a slope. J Fluid Mech 374, 305-333.

Isshiki Y (1980) Nitrogen components of caecal contents in fasted chickens. Jpn J Zootech Sci 51, 12-16.

Jaroni D, Scheideler SE, Beck MM \& Wyatt C (1999) The effect of dietary wheat middlings and enzyme supplementation II: apparent nutrient digestibility, digestive tract size, gut viscosity, and gut morphology in two strains of leghorn hens. Poult Sci 78, 1664-1674.

Jeffrey DJ \& Acrivos A (1976) The rheological properties of suspensions of rigid particles. Am Inst Chem Eng $J$ 22, 417-430.

Karasawa Y (1989) Effect of colostomy on nitrogen nutrition in the chicken fed a low protein diet plus urea. J Nutr 119, $1388-1391$.

Klasing KC (2000) Digestion of food. In Comparative Avian Nutrition, pp. 36-70 New York: CAB International.

Lentle RG, Stafford KJ, Kennedy MS \& Haslett SJ (2002) Rheological properties of digesta suggest little radial or axial mixing in the forestomach of the tammar (Macropus eugenii) and the parma (Macropus parma) wallaby. Physiol Biochem Zool 75, 572-582.

Razdan A \& Pettersson D (1996) Hypolipidaemic, gastrointestinal and related responses of broiler chickens to chitosans of different viscosity. Br Poult Sci 76, 387-397.

Saraf DN \& Khullar SD (1975) Some studies on the viscosity of settling suspensions. Can J Chem Eng 53, 449-452. 
Sokal RR \& Rohlf JS (1995) Biometry, 3rd ed., pp. 820-825. San Francisco, CA: Freeman.

Takahashi T \& Sakata T (2002) Large particles increase viscosity and yield stress of pig cecal contents without changing basic viscoelastic properties. J Nutr 132, 1026-1030.

Takahashi T, Sakata T, Yamanaka N \& Ogawa N (2001) Effects of dietary particles on the viscosity of gut contents and intestinal tissue weight. Ann Nutr Metab 45, Suppl. 1, 78.

Takahashi T, Sakata T, Yamanaka N \& Ogawa N (2003) Influence of solid particles on the viscous properties of intestinal contents and intestinal tissue weight in rats. J Jpn Soc Nutr Food Sci 56, 199-205.

Van der Klis JD, Verstegen MW \& van Voorst A (1993) Effect of a soluble polysaccharide (carboxy methyl cellulose) on the absorption of minerals from the gastrointestinal tract of broilers. Br Poult Sci 34, 985-997.

Yoon WB \& McCarthy KL (2002) Rheology of yogurt during pipe flow as characterized by magnetic resonance imaging. $J$ Texture Stud 33, 431-444.

Zar JH (1999) Biostatistical Analysis, 4th ed., New Jersey: Prentice-Hall Inc.

Zimeri JE \& Kokini JL (2003) Rheological properties of inulinwaxy maize starch systems. Carbohydr Polym 52, 67-85.

Zubair AK, Forsberg CW \& Leesson S (1996) Effect of dietary fat, fiber, and monensin on caecal activity in turkeys. Poult Sci 75, 891-899. 